\title{
The cover time of random geometric graphs
}

\author{
Colin Cooper* $\quad$ Alan Frieze ${ }^{\dagger}$
}

August 19, 2009

\begin{abstract}
We study the cover time of random geometric graphs. Let $I(d)=[0,1]^{d}$ denote the unit torus in $d$ dimensions. Let $D(x, r)$ denote the ball (disc) of radius $r$. Let $\Upsilon_{d}$ be the volume of the unit ball $D(0,1)$ in $d$ dimensions. A random geometric graph $G=$ $G(d, r, n)$ in $d$ dimensions is defined as follows: Sample $n$ points $V$ independently and uniformly at random from $I(d)$. For each point $x$ draw a ball $D(x, r)$ of radius $r$ about $x$. The vertex set $V(G)=V$ and the edge set $E(G)=\{\{v, w\}: w \neq v, w \in D(v, r)\}$. Let $G(d, r, n), d \geq 3$ be a random geometric graph. Let $C_{G}$ denote the cover time of a simple random walk on $G$. Let $c>1$ be constant, and let $r=\left(c \log n /\left(\Upsilon_{d} n\right)\right)^{1 / d}$. Then whp the cover time satisfies

$$
C_{G} \sim c \log \left(\frac{c}{c-1}\right) n \log n
$$
\end{abstract}

\section{Introduction}

Let $G=(V, E)$ be a connected graph with $|V|=n$ vertices, and $|E|=m$ edges. In a simple random walk $W$ on a graph $G$, at each step, a particle moves from its current vertex to a randomly chosen neighbour. For $v \in V$, let $C_{v}$ be the expected time taken for a simple random walk starting at $v$ to visit every vertex of $G$. The vertex cover time $C_{G}$ of $G$ is defined as $C_{G}=\max _{v \in V} C_{v}$. The (vertex) cover time of connected graphs has been extensively studied. It is a classic result of Aleliunas, Karp, Lipton, Lovász and Rackoff [2] that $C_{G} \leq 2 m(n-1)$. It was shown by Feige [15], [16], that for any connected graph $G$, the cover time satisfies

*Department of Computer Science, King's College, University of London, London WC2R 2LS, UK

$\dagger$ Department of Mathematical Sciences, Carnegie Mellon University, Pittsburgh PA15213, USA. Supported in part by NSF grant CCF0502793. 
$(1-o(1)) n \log n \leq C_{G} \leq(1+o(1)) \frac{4}{27} n^{3}$. As an example of a graph achieving the lower bound, the complete graph $K_{n}$ has cover time determined by the Coupon Collector problem. The lollipop graph consisting of a path of length $n / 3$ joined to a clique of size $2 n / 3$ gives the asymptotic upper bound for the cover time.

A few words on notation. Results on random graphs are always asymptotic in $n$, the size of the vertex set. The notation $A_{n} \sim B_{n}$ means that $\lim _{n \rightarrow \infty} A_{n} / B_{n}=1$, and whp (with high probability) means with probability tending to 1 as $n \rightarrow \infty$. Poly-log factors are suppressed in $\tilde{O}, \tilde{\Omega}$.

In a series of papers, we have studied the cover time of various models of a random graph. These results can be summarized as follows:

- [10] If $p=c \log n / n$ and $c>1$ then whp $C_{G_{n, p}} \sim c \log \left(\frac{c}{c-1}\right) n \log n$.

- [11] Let $c>1$ and let $x$ denote the solution in $(0,1)$ of $x=1-e^{-c x}$. Let $X_{g}$ be the giant component of $G_{n, p}, p=c / n$. Then whp $C_{X_{g}} \sim \frac{c x(2-x)}{4(c x-\log c)} n(\log n)^{2}$.

- [8] Let $G_{n, r}$ denote a random $r$-regular graph on vertex set $[n]$ with $r \geq 3$ then whp $C_{G_{n, r}} \sim \frac{r-1}{r-2} n \log n$.

- [9] Let $G_{m}(n)$ denote a preferential attachment graph of average degree $2 m$ then whp $C_{G_{m}} \sim \frac{2 m}{m-1} n \log n$.

- [12] Let $D_{n, p}$ denote a random digraph with independent edge probability $p$. If $p=$ $c \log n / n$ and $c>1$ then whp $C_{D_{n, p}} \sim c \log \left(\frac{c}{c-1}\right) n \log n$.

Let $I$ denote the unit interval $[0,1]$ and let $I(d)=[0,1]^{d}$ denote the unit torus in $d$ dimensions. We use the torus for convenience, to avoid boundary effects. Let $D(x, r)$ denote the ball (disc) of radius $r$, and thus

$$
D(x, r)=\left\{y \in I(d): \sum_{i=1}^{d} \min \left\{\left|x_{i}-y_{i}\right|^{2},\left|x_{i}-\left(1+y_{i}\right)\right|^{2}\right\} \leq r^{2}\right\} .
$$

Let $\Upsilon_{d}$ be the volume of the unit ball $D(0,1)$ in $d$ dimensions. Thus

$$
\Upsilon_{d}=\left(\pi^{d / 2}\right) / \Gamma(d / 2+1)= \begin{cases}\frac{\pi^{k}}{k !} & d=2 k, \text { even } \\ \frac{2^{d} k ! \pi^{k}}{d !} & d=2 k+1, \text { odd }\end{cases}
$$

A random geometric graph $G=G(d, r, n)$ in $d$ dimensions is defined as follows: Sample $n$ points $V$ independently and uniformly at random from $I(d)$. For each point $x$ draw a 
ball $D(x, r)$ of radius $r$ about $x$. The vertex set $V(G)=V$ and the edge set $E(G)=$ $\{\{v, w\}: w \neq v, w \in D(v, r)\}$

Geometric graphs are widely used as models of ad-hoc wireless networks [18], [19], [24] in which each transmitter has transmission radius $r$ and can only communicate with other transmitters within that radius. In the simplest model of a random geometric graph, the $n$ points representing transmitters, are distributed uniformly at random (uar) in the unit square. Any other point $v$ within the circle radius $r$ centered at a transmitter $u$, is joined to $u$ by an edge.

If $r \geq \sqrt{c \log n /(\pi n)}, c>1$, then the graph $G(d, r, n)$ is connected whp [18, 21], and the cover time of $G$ is well defined. Avin and Ercal [3] consider the cover time of geometric graphs in the case $d=2$, and prove the following theorem.

Theorem 1. If $G=G(2, r, n)$ and $r^{2}>\frac{8 \log n}{n}$ then whp

$$
C_{G}=\Theta(n \log n)
$$

They also indicate that this result can be generalized to $d \geq 3$. In this paper we consider $d \geq 3$ and replace $\Theta(n \log n)$ by an asymptotically correct constant:

Theorem 2. Let $G(d, r, n), d \geq 3$ be a random geometric graph. Let $c>1$ be constant, and let $r=\left(\frac{c \log n}{\Upsilon_{d} n}\right)^{1 / d}$. Then whp

$$
C_{G} \sim c \log \left(\frac{c}{c-1}\right) n \log n
$$

As $c$ increases, the RHS of (1) is asymptotic to $n \log n$. It will be clear that we can allow $c \rightarrow \infty$ in our analysis and obtain this estimate rigorously. We find it convenient however just to deal with the case of $c$ constant.

\section{Structure of the paper}

In common with [8]-[12] the paper relies on the first visit time lemma (Lemma 11) to estimate the cover time. Using this lemma requires us to estimate $R_{v}$, the expected number of returns to vertex $v$ within time $T$ made by a random walk $\mathcal{W}_{v}$ which starts from vertex $v$. Here $T$ (see equation (8)) is a bound on the time needed for a random walk to get sufficiently close in distribution to the steady state to apply Lemma 11.

Normally calculations of $R_{v}$ rely on symmetry or locally tree-like structure of the graph, at least for most vertices. Moreover there is often a large set of vertices $v$ for which the ratio 
$d(v) / R_{v}$ is clearly minimum. These vertices are hardest to reach and determine the cover time.

It turns out that provided the conditions of Theorem 2 are satisfied, then $R_{v}=1+o(1)$ for all $v$, but the main difficulty is proving this fact, and most of the paper is devoted to this task. Several technical difficulties arise, mainly due to the high edge density between neighbours of a vertex, the lack of homogeneity in the graph itself and the variation in spatial distribution of vertices within the $d$-dimensional unit cube $I(d)$. This last point is especially troublesome when the parameter $c$ in the disc-radius tends to 1 (the connectivity threshold).

To establish the result, we were obliged to prove some very precise results on connectivity properties of the graph, based on a dissection of the unit cube $I(d)$ into sub-cubes. Most of Section 2, is devoted to proving that $G$ contains a large $d$-dimensional connected grid-like structure $\Gamma$ of vertices in "heavy" sub-cubes. All other vertices are connected to $\Gamma$ by short paths. (Here a cube is heavy if it contains at least $a \log n$ vertices, for some $a>0$ ). This proof causes some technical difficulties, because we want to have $c$ arbitrarily close to 1 and not just sufficiently large.

Having done this, in Section 4 we estimate the mixing time $T$. This is not a new result, but is included for completeness. The proof is straightforward once we have proved the existence of $\Gamma$. The value of $T=\tilde{O}\left(n^{2 / d}\right)$ is much larger than in papers [8]-[12], where it is polylog $(n)$. This means there is more time for the walk to return to $v$ and so verifying $R_{v}=1+o(1)$ is non-trivial. This takes up Section 5. The analysis here is specific to the paper, using effective resistance, Raleigh's theorem on edge deletion and Pólya's theorem on random walks on $R^{d}, d \geq 3$. Indeed there are several difficulties in relating properties of the walk to the grid-like subgraph we focused on in Section 2 (see Lemma 13, and subsequent sub-lemmas).

Having established $R_{v}=1+o(1)$ we can apply Lemma 11 to obtain an upper bound on cover time. This is Section 6.1. When we come to prove the lower bound in Section 6.2, we use the Chebyshev inequality, as in previous papers. It turns out to be much more complicated to apply, especially for $c$ close to one. We had to resort to the idea of coupling the construction of $G$ along with the construction of the random walk $\mathcal{W}_{v}$ and then applying the Berry-Esseen inequality.

\section{Some properties of $G=G(d, r, n)$}

The degree, $d(v)$, of vertex $v \in V$ is binomially distributed as $\operatorname{Bin}(n-1, p)$, where $p=c \log n / n$ is the volume of $D(v, r)$. It is a simple matter to show using the Chebychev inequality that whp the number of edges $m$ of $G$ satisfies

$$
m \sim \frac{1}{2} c n \log n .
$$


We next give some crude bounds on vertex degree.

Lemma 3. Let $n p=c \log n$. For $c>1$ there exists a constant $c_{0}>0$ such that $\mathbf{w h p}$

$$
c_{0} \log n \leq d(v) \leq \Delta_{0}=(c+10) \log n \quad \text { for all } v \in V .
$$

Proof For $c_{0}$ sufficiently small,

$$
\begin{aligned}
\operatorname{Pr}\left(\exists v: d(v) \leq c_{0} \log n\right) & \leq n \operatorname{Pr}\left(\operatorname{Bin}(n-1, p) \leq c_{0} \log n\right) \\
& =n \sum_{k=0}^{c_{0} \log n}\left(\begin{array}{c}
n-1 \\
k
\end{array}\right) p^{k}(1-p)^{n-1-k} \\
& \leq 2 n\left(\frac{n e}{c_{0} \log n}\right)^{c_{0} \log n}\left(\frac{c \log n}{n}\right)^{c_{0} \log n} n^{-c+o(1)} \\
& =n^{-\left(c-1-c_{0} \log \left(c e / c_{0}\right)-o(1)\right)} \\
& =o(1) .
\end{aligned}
$$

On the other hand,

$$
\begin{aligned}
\operatorname{Pr}\left(\exists v: d(v) \geq \Delta_{0}\right) & \leq n \operatorname{Pr}\left(\operatorname{Bin}(n-1, p) \geq \Delta_{0}\right) \\
& \leq n \sum_{k \geq \Delta_{0}}\left(\begin{array}{l}
n \\
k
\end{array}\right)\left(\frac{c \log n}{n}\right)^{k}\left(1-\frac{c \log n}{n}\right)^{n-k} \\
& \leq 2 n\left(\begin{array}{c}
n \\
\Delta_{0}
\end{array}\right)\left(\frac{c \log n}{n}\right)^{\Delta_{0}}\left(1-\frac{c \log n}{n}\right)^{n-\Delta_{0}} \\
& \leq 3 n\left(\frac{n e}{\Delta_{0}}\right)^{\Delta_{0}}\left(\frac{c \log n}{n}\right)^{\Delta_{0}} n^{-c} \\
& =3 n^{1-c-(c+10) \log ((c+10) /(c e))} \\
& =o(1) .
\end{aligned}
$$

Let $D(k)$ denote the number of vertices $v$ with $d(v)=k$ in $G$, and let $\bar{D}(k)$ be the expected number. Thus

$$
\bar{D}(k)=n\left(\begin{array}{c}
n-1 \\
k
\end{array}\right) p^{k}(1-p)^{n-1-k} \leq \frac{2}{n^{c-1}}\left(\frac{n e p}{k}\right)^{k} .
$$

Let

$$
I_{c}=\left[c_{0} \log n, \Delta_{0}\right]
$$

where $\Delta_{0}=(c+10) \log n$. The previous lemma shows that whp all vertex degrees lie in $I_{c}$. The next lemma gives bounds the value of $D(k)$ in terms of $\bar{D}(k)$ for various ranges of $k \in I_{c}$. 
Lemma 4. Let

$$
\begin{aligned}
& K_{0}=\left\{k \in I_{c}: \bar{D}(k) \leq(\log n)^{-2}\right\} \\
& K_{1}=\left\{k \in I_{c}:(\log n)^{-2} \leq \bar{D}(k) \leq(\log n)^{2}\right\} . \\
& K_{2}=I_{c} \backslash\left(K_{0} \cup K_{1}\right) .
\end{aligned}
$$

\section{Then, whp}

(a) For $k \in K_{0}, D(k)=0$.

For $k \in K_{1}, D(k) \leq(\log n)^{4}$, and

$$
\min \left\{k \in K_{1}\right\} \geq(\log n)^{1 / 2} \quad \text { and } \quad\left|K_{1}\right|=O(\log \log n) .
$$

If $k \in K_{2}$ then $\frac{1}{2} \bar{D}(k) \leq D(k) \leq 2 \bar{D}(k)$.

(b) Let $\gamma_{c}=(c-1) \log (c /(c-1))$. Let $k_{1}=\lceil(c-1) \log n\rceil$, and let $S_{1}=\left\{v: d(v)=k_{1}\right\}$, then

$$
\left|S_{1}\right|=\left(n e p / k_{1}\right)^{k_{1}} n^{1-c+o(1)}=n^{\gamma_{c}+o(1)} .
$$

Proof An identical calculation is made in [10] for the degree sequence of the random graph $G_{n, p}$.

The remainder of this section is devoted to proving that vertices of $G$ which are close spatially in $I(d)=[0,1]^{d}$, are close in edge distance in $G$. To do this we partition $I(d)$ into sub-cubes of various (appropriate) sizes, and examine the structure of the graph within and between the sub-cubes. This partition approach is used in many of the proofs throughout this paper.

Let

$$
h_{a}=\epsilon_{a} r \text { and } h_{b}=L_{1} h_{a}
$$

where $\epsilon_{a} \leq 1 / 4 d$ is a small positive constant. We assume that $\ell_{a}=1 / h_{a}$ is an even integer and $L_{1}$ is a large odd integer constant which divides $\ell_{a}$, and thus $\ell_{a} / L_{1}$ is even. We will assume that

$$
L_{1} \epsilon_{a} \gg 1
$$

so that a ball of radius $r$ fits well into a cube of side $L_{1} h_{a}$.

The size of $L_{1}$ is constrained by the inequalities (6) and (7); where $\Gamma_{d}$ is given by (4) and the parameter $L$ of inequality (7) satisfies (5).

We partition $I(d)$ into grids $K_{a}, K_{b}$ where $K_{a}, K_{b}$ are made up of cubes of side $h_{a}, h_{b}$ and $K_{a}$ is a refinement of $K_{b}$. We assume that $\epsilon_{a}$ is small enough so that if $x, y$ are in $K_{a}$-cubes that share a $(d-1)$-dimensional face then $x, y$ are adjacent in $G$. 
Let $B_{1}, B_{2}, \ldots, B_{M}, M=\Omega(n / \log n)$ be an enumeration of the $K_{b}$-cubes. The aim of the next few lemmas is to show that whp we can choose a $K_{a}$-cube $A_{i}$ inside each $B_{i}$ such that each $A_{i}$ contains $\Omega(\log n)$ members of $V$ (we say $A_{i}$ is a heavy sub-cube). Furthermore, if $B_{i}, B_{j}$ share a $(d-1)$-dimensional face, then there is a sequence of $3 L_{1}+1$ heavy $K_{a}$-cubes $X_{0}, X_{1}, \ldots X_{3 L_{1}}$ such that (i) $X_{0}=A_{i}$ and $X_{3 L_{1}}=A_{j}$ and (ii) $X_{i}, X_{i+1}$ share a $(d-1)$ dimensional face for $0 \leq i<3 L_{1}$. $L_{1}$ is some sufficiently large constant. In other words, we find a large $d$-dimensional grid-like structure with vertices represented by the $A_{i}$ and edges represented by a sequence of $3 L_{1}+1$ heavy cubes. Furthermore, every other vertex will be within $O(1)$ distance of this "grid".

Each $K_{a}$-cube is labeled by a $d$-tuple in $\left[\ell_{a}\right]^{d}$. Given a $K_{b}$-cube $B$ we define a line of $B$ to be a set of $L_{1} K_{a}$-cubes of $B$, where the labels are constant except for exactly one index. A slice of $B$ is a set of $L_{1}^{d-1} K_{a}$-cubes of $B$, where the labels are constant on exactly one index. A slice is extreme if contains a $(d-1)$-dimensional face of $B$. Given a line of $B$, its two ends are the two $K_{a}$-cubes lying in extreme slices.

If we fix a $K_{a}$-cube $A$, then the number of points in $V$ that are in $A$ is distributed as $\operatorname{Bin}(n, \alpha \log n / n)$ where $\alpha=c \epsilon_{a}^{d} / \Upsilon_{d}$. A cube is light if it contains fewer than $\epsilon_{\ell} \alpha \log n$ points in $V$ where $\epsilon_{\ell} \leq c_{0} / 2 c$ is a small constant, otherwise it is heavy. If $C$ is an arbitrary union of $K_{a}$-cubes $A_{1}, A_{2}, \ldots, A_{k}$ then heavy $(C)=\left\{A_{i}: A_{i}\right.$ is heavy $\}$.

Given a $K_{a}$-cube $A$, let $K_{L}(A)$ be a cube of side $L h_{a}$ with $A$ at its centre (assuming that $L$ is an odd integer). Consider $K_{L}(A)$ to be partitioned into $L^{d} K_{a}$-cubes.

Lemma 5. Suppose that $L=O(1)$. Let

$$
\Gamma_{d}=20 \Upsilon_{d} \epsilon_{a}^{-d} .
$$

Then whp there does not exist a $K_{a}$-cube $A$ such that $K_{L}(A)$ contains $\Gamma_{d}$ light $K_{a}$-cubes

Proof The number of points of $V$ in a set of $t K_{a}$-cubes is distributed as the binomial $\operatorname{Bin}(n, t \alpha \log n / n)$. If they are all light then this number is less than $t \alpha \log n / 2$. Using Chernoff bounds, we see that the probability that $K_{L}(A)$ contains at least $t \geq \Gamma_{d}$ light $K_{a}$-cubes is at most

$$
\left(\begin{array}{c}
L^{d} \\
t
\end{array}\right) e^{-t \alpha \log n / 8} \leq\left(L^{d} n^{-c \epsilon_{\alpha}^{d} /\left(8 \Upsilon_{d}\right)}\right)^{t} \leq n^{-2} .
$$

There are $O(n) K_{a}$-cubes and the claim follows.

We use the following result, which is part of Lemma 9.9 of Penrose [21]: Let $B_{Z}(n)=[n]^{d}$ and let $A$ be a subset of $B_{Z}(n)$. We assume a graph structure with vertices $[n]^{d}$, and where two vertices are adjacent if their Hamming distance is one. The external vertex boundary $\partial_{B(n)}^{+} A$ is the set of vertices in $B_{Z}(n) \backslash A$ which are adjacent to some $x \in A$. 
Lemma 6. If $A \subseteq[n]^{d}$ and $|A| \leq 2 n^{d} / 3$ then

$$
\left|\partial_{B(n)}^{+} A\right| \geq(2 d)^{-1}\left(1-(2 / 3)^{1 / d}\right)|A|^{(d-1) / d} .
$$

Fix a cube $C$ that is the union of $K_{a}$-cubes and is of side $L h_{a}, L=O(1)$. Consider the graph $H_{C}$ with vertex set heavy $(C)$ and where two vertices of $H_{C}$ are adjacent if the corresponding cubes share a $(d-1)$-dimensional face. Let $\kappa_{1}\left(H_{C}\right)$ denote the size of the largest component of $H_{C}$. We a somewhat loosely refer to this as "the largest component of $C$ ".

\section{Lemma 7. Whp}

$$
\kappa_{1}\left(H_{C}\right) \geq L^{d}-\gamma \Gamma_{d}^{2}
$$

for some $\gamma=\gamma(d) \geq 1$.

Proof It follows from Lemma 5 that $\left|V\left(H_{C}\right)\right|=\mid$ heavy $(C) \mid \geq L^{d}-\Gamma_{d}$. Let $W_{1}, W_{2}, \ldots, W_{s}$ be the components of $H_{C}$. Suppose that some $r$-subset of components $W_{1}, \ldots, W_{r}$ satisfies $W=$ $\bigcup_{i=1}^{r} W_{i}$ where $|W|=w \leq L^{d} / 2$. Lemma 6 implies that $W$ has at least $a_{1} w^{(d-1) / d}$ neighbours for some $a_{1}=a_{1}(d)$. As these must all be light this implies that $\Gamma_{d} \geq a_{1} w^{(d-1) / d} \geq a_{1} w^{1 / 2}$ and the claim follows.

Recall that $h_{b}=L_{1} h_{a}$ defines the side length of the $K_{b}$-cubes. Let $A$ be the centre $K_{a}$-cube of a $K_{b}$-cube $B$. We introduce a new quantity $L$ and define $K_{L}^{*}(B)$, the $L$-centre of $B$, to be $K_{L}(A)$. If $F$ is an extreme slice of $B$, then define its $L$-centre as follows: If $X$ is the centre $K_{a}$-cube of $F$ then $K_{L}^{*}(F)=F \cap K_{L}(X)$.

A line $\Lambda$ of $B$ containing a cube $\hat{A} \in K_{L}^{*}(B)$ is good if it satisfies the following conditions:

1. All its $K_{a}$-cubes are in $\kappa_{1}\left(H_{B}\right)$.

2. Let $A_{1}, A_{2}$ be the $K_{a}$-cubes at the ends of $\Lambda$. Let $F_{i}$ be the extreme slice containing $A_{i}$. Let $F_{i}^{*}=K_{L}^{*}\left(F_{i}\right)$. Let $H_{i}^{*}$ be the sub-graph of $H_{B}$ induced by $F_{i}^{*}$. We require that $A_{i} \in \kappa_{1}\left(H_{i}^{*}\right)$ for $i=1,2$.

We say that a $K_{a}$-cube $\hat{A}$ is good if $\hat{A} \in K_{L}^{*}(B) \cap \kappa_{1}\left(H_{B}\right)$ and if the $d$ lines through $\hat{A}$ are good. A good cube $\hat{A}$ is useable if all $K_{a}$-cubes within distance 10 of $\hat{A}$ are good.

Lemma 8. $K_{L}^{*}(B)$ contains at least $L^{d}-(2 d)^{11} L \gamma \Gamma_{d}^{2}$ useable cubes, whp.

Proof Let $B$ be a $K_{b}$-cube. Every useable sub-cube of $B$ lies in $\kappa_{1}\left(H_{B}\right)$. The number of lines containing a light cube of $B$ is at most $d \Gamma_{d}$. The number of lines with an end-cube in 
the $L$-centre of its face that violate Condition 2 of goodness is at most $2 d \times \gamma \Gamma_{d-1}^{2}$. (We have applied Lemma 7 to the $F_{i}^{*}$ of this condition).

Each cube has at most $(2 d)^{10}$ cubes within distance 10 . So the number of non-useable cubes in the $L$-centre is at most $L(2 d)^{10}\left(d \Gamma_{d}+2 d \gamma \Gamma_{d-1}^{2}\right)$.

We make $L$ large enough so that

$$
L^{d}-(2 d)^{11} L \gamma \Gamma_{d}^{2}>L^{d} / 2
$$

and then we can apply Lemma 8 to show there are many useable cubes. We also assume that

$$
L_{1} \geq L^{d}
$$

Finally, Lemma 10 below, requires the following lower bound on $L_{1}$ :

$$
L_{1} \geq 3^{d}\left(\Gamma_{d}+\gamma \Gamma_{d}^{2}\right) / \epsilon_{a}
$$

Let $B_{1}, B_{2}, \ldots, B_{M}, M=\Omega(n / \log n)$ be an enumeration of the $K_{b}$-cubes. Our grids define bipartite graphs, this is why we chose $\ell_{a}, \ell_{a} / L_{1}$ even. Thus each cube will have a parity, with neighbouring cubes having different parity. Similarly, the $K_{a}$ sub-cubes of each $B_{i}$ have a parity. We choose a useable cube $A_{i}$ in each $B_{i}, i=1,2, \ldots, M$, where $A_{i}$ is chosen to have the same parity as $B_{i}$.

In what follows, by a path, we mean a sequence of $K_{a}$-cubes (the path vertices) with consecutive cubes sharing a $(d-1)$-face.

Lemma 9. If $K_{b}$-cubes $B_{i}, B_{j}$ share $a(d-1)$-dimensional face, then there is a path $P(i, j)$ of length $3 L_{1}$ of heavy cubes joining $A_{i}$ and $A_{j}$, where $A_{i}, A_{j}$ are useable cubes, as defined above. These paths are pair-wise internally vertex disjoint.

Proof First define a path $Q(i, j)$ as follows: Start at $A_{i}$ and follow the line $\Lambda_{i}$ of cubes to the face separating $B_{i}$ and $B_{j}$. Suppose this ends in the cube $S_{i}$. Do the same for $A_{j}$. Now choose a cube $S$ in $\kappa_{1}\left(F_{i}^{*}\right)$ that shares a $(d-1)$-face with a cube $S^{\prime}$ in $\kappa_{1}\left(F_{j}^{*}\right)$. This is possible if $L$ is large enough. $Q(i, j)$ consists of $\Lambda_{i}$, then a path from $S_{i}$ to $S$ inside $\kappa_{1}\left(F_{i}^{*}\right)$, then the edge to $S^{\prime}$, then a path from $S^{\prime}$ to $S_{j}$ inside $\kappa_{1}\left(F_{j}^{*}\right)$ and finally the line $\Lambda_{j}$ in the direction $S_{j}$ to $A_{j}$. The $Q(i, j)$ are internally vertex disjoint by construction and have odd length at most $2 L_{1}+L^{d} \leq 3 L_{1}$, from (6). The odd length follows from the fact that the parities of $B_{i}, B_{j}$ differ. We now add to $Q(i, j)$ to bring its length up to $3 L_{1}$. Suppose $Q(i, j)$ has length $3 L_{1}-2 t$ where $t$ is a positive integer. Suppose that $Q(i, j)$ begins with cubes $A_{i}, S_{1}, S_{2}$. We will replace the edge $\left(S_{1}, S_{2}\right)$ by a path of length $2 t+1$. Suppose that we number the $2 d$ coordinate directions as $\pm 1, \pm 2, \ldots, \pm d$. Suppose that $Q(i, j)$ starts in direction $+k$. If $t=1$ 
we replace $\left(S_{1}, S_{2}\right)$ by $\left(S_{1}, T_{1}, T_{2}, S_{2}\right)$ where $T_{l}$ is the neighbour of $S_{l}$ in direction $+(k+1)$. Otherwise, let $T_{l}^{\prime}$ be the neighbour of $T_{l}$ in the direction $+(k+1)$. We can now construct a path $S_{1}, T_{1}, T_{1}^{\prime}, T_{2}^{\prime}, P_{h}, T_{2}, S_{2}$ where $P_{h}$ is a path of length $2 h+1,0 \leq h \leq\left(L_{1}-L\right) / 2$. The path $P_{h}$ goes out from $T_{2}^{\prime}$ for $h$ steps in direction $+k$, then makes a step in direction $-(k+1)$ and then returns to $T_{2}$ via $h$ steps in direction $-k$. This path will only use heavy cubes, by the definition of useable. This suffices for $2 h+4=2 t$ provided $t \leq L_{1}-L+2$. For larger $t$ we will have to go out further in the direction $+(k+1)$ and use two or three paths in the direction $+k$. Our choice of directions will maintain disjointness.

We now consider points that do not lie in a cube of $\kappa_{1}\left(H_{B}\right)$ for any $B$ in the $K_{b}$ dissection of $I(d)$.

Lemma 10. For $v \in V$, let $C_{v}^{(b)}$ be the $K_{b}$-cube containing $v$ in the $K_{b}$ dissection of $I(d)$ and let $C_{v}^{(a)}$ be the $K_{a}$-cube containing $v$ in the $K_{a}$ dissection of $C_{v}^{(b)}$. Then, there exists a $K_{b}$-cube $B_{i}$ such that

(a) $v$ is at $G$-distance $\leq 3^{d}\left(\Gamma_{d}+\gamma \Gamma_{d}^{2}\right)$ from $\kappa_{1}\left(H_{B_{i}}\right)$.

(b) $v$ is at $G$-distance $O(1)$ from any point $w \in V$ in $B_{i}$.

Proof Let $\ell=3^{d}\left(\Gamma_{d}+\gamma \Gamma_{d}^{2}\right)$. Fix $v \in V$ lying in the cube $C_{v}^{(a)}$ and let $P=\left(v_{0}=\right.$ $\left.v, v_{1}, \ldots, v_{k}\right)$ be a shortest path in $G$ from $v$ to a point in an $H_{B_{i}}$. Here we are assuming that $G$ is connected, which it is whp.

We can assume that the $K_{a}$-cubes that contain these $v_{i}$ are distinct, otherwise the path can be shortened. Given (7) we can see that $k \leq \ell$. Indeed, if $\ell<k$ then (7) implies that the subpath $\left(v_{0}, v_{1}, \ldots, v_{\ell}\right)$ stays inside the $\leq 3^{d} K_{b}$-cubes that touch $C_{v}^{(b)}$. It follows from Lemmas 5 and 7 that at least one of $K_{a}$-cubes containing one of the $v_{1}, v_{2}, \ldots, v_{\ell}$ is in the largest heavy component of its $K_{b}$-cube. This verifies $k<\ell$ and also proves part (a). Once we reach the largest component of $B_{i}$, we can reach a vertex in $A_{i}$ in $O(1)$ steps, which proves part (b).

\section{Estimating first visit probabilities}

In this section we describe the main analytical tool we use to compute the cover time. We use the approach of $[8,9,11,12]$. Let $G$ denote a fixed connected graph, and let $u$ be some arbitrary vertex from which a walk $\mathcal{W}_{u}$ is started. Let $\mathcal{W}_{u}(t)$ be the vertex reached at step $t$, let $P$ be the matrix of transition probabilities of the walk, and let $P_{u}^{(t)}(v)=\operatorname{Pr}\left(\mathcal{W}_{u}(t)=v\right)$. Let $\pi$ be the steady state distribution of the random walk $\mathcal{W}_{u}$. Let $\pi_{v}=\pi(v)$ denote the 
stationary distribution of the vertex $v$. For an unbiased ergodic random walk on a graph $G$ with $m=m(G)$ edges, $\pi_{v}=\frac{d(v)}{2 m}$, where $d(v)$ denotes the degree of $v$ in $G$.

Let $d(t)=\max _{u, x \in V}\left|P_{u}^{(t)}(x)-\pi_{x}\right|$, and let $T$ be such that, for $t \geq T$

$$
\max _{u, x \in V}\left|P_{u}^{(t)}(x)-\pi_{x}\right| \leq n^{-3} .
$$

It follows from e.g. Aldous and Fill [1] that $d(s+t) \leq 2 d(s) d(t)$ and so for $k \geq 1$,

$$
\max _{u, x \in V}\left|P_{u}^{(k T)}(x)-\pi_{x}\right| \leq \frac{2^{k-1}}{n^{3 k}} .
$$

Now fix $u \neq v \in V$. Next, let $r_{t}=\operatorname{Pr}\left(\mathcal{W}_{v}(t)=v\right)$ be the probability that this walk returns to $v$ at step $t$. Let

$$
R_{T}(z)=\sum_{j=0}^{T-1} r_{j} z^{j}
$$

For a large constant $K>0$, let

$$
\lambda=\frac{1}{K T}
$$

For $t \geq T$, let $\mathcal{A}_{t}(v)$ be the event that $\mathcal{W}_{u}$ does not visit $v$ in steps $T, T+1, \ldots, t$ The vertex $u$ will have to be implicit in this definition. The following was proved in [11].

Lemma 11. Suppose that

(a) For some constant $\theta>0$, we have

$$
\min _{|z| \leq 1+\lambda}\left|R_{T}(z)\right| \geq \theta
$$

(b) $T \pi_{v}=o(1)$ and $T \pi_{v}=\Omega\left(n^{-2}\right)$.

Let

$$
p_{v}=\frac{\pi_{v}}{R_{T}(1)\left(1+O\left(T \pi_{v}\right)\right)},
$$

where $R_{T}(1)$ is from (10).

Then for all $t \geq T$,

$$
\operatorname{Pr}\left(\mathcal{A}_{t}(v)\right)=\frac{\left(1+O\left(T \pi_{v}\right)\right)}{\left(1+p_{v}\right)^{t}}+O\left(T e^{-\lambda t / 2}\right)
$$

The evaluation of $R_{T}(z)$ at $z=1$ occurs frequently in our calculations in this paper. For the rest of the paper $u, v$ will not be fixed and it is appropriate to replace the notation $R_{T}(1)$ by something dependent on $v$. We use the notation $R_{v}$. Our next two tasks are to estimate $T$ and the $R_{v}$. 


\section{Mixing time of the random walk}

We need two basic results on mixing times. These results and the appropriate related definitions can be found in e.g. Lovász [20], and Sinclair [22] respectively.

First let $\lambda_{\max }$ be the second largest eigenvalue of the transition matrix $P$. Then,

$$
\left|P_{u}^{(t)}(x)-\pi_{x}\right| \leq\left(\frac{\pi_{x}}{\pi_{u}}\right)^{1 / 2} \lambda_{\max }^{t} \leq\left((c+10) / c_{0}\right)^{1 / 2} \lambda_{\max }^{t} .
$$

Next, for each $x \neq y \in V$ let $\gamma_{x y}$ be a canonical path from $x$ to $y$ in $G$. Then, we have that

$$
\lambda_{\max } \leq 1-\frac{1}{\rho}
$$

where

$$
\rho=\max _{e=\{x, y\} \in E(G)} \frac{1}{\pi(x) P(x, y)} \sum_{\gamma_{a b} \ni e} \pi(a) \pi(b)\left|\gamma_{a b}\right|,
$$

and $\left|\gamma_{a b}\right|$ is the length of the canonical path $\gamma_{a b}$ from $a$ to $b$.

Consider the $K_{b}$-grid of Section 2. It will help to fix a collection of points $x_{i} \in A_{i} \cap V$ for $i=1,2, \ldots, M$.

\section{Lemma 12.}

$$
1-\lambda_{\max }=\tilde{\Omega}\left(n^{-2 / d}\right) .
$$

Proof We first define canonical paths between the $x_{i}$. We can in a natural way express $x_{i}=y\left(j_{1}, j_{2}, \ldots, j_{d}\right)$ where $0 \leq j_{t}<1 / h_{b}$ for $1 \leq t \leq d$. The path from $y\left(j_{1}, j_{2}, \ldots, j_{d}\right)$ to $y\left(k_{1}, k_{2}, \ldots, k_{d}\right)$ goes

$$
\begin{aligned}
& y\left(j_{1}, j_{2}, \ldots, j_{d}\right) \leadsto y\left(j_{1}+1, j_{2}, \ldots, j_{d}\right) \leadsto \cdots, y\left(k_{1}, j_{2}, \ldots, j_{d}\right) \rightsquigarrow \\
& y\left(k_{1}, j_{2}+1, \ldots, j_{d}\right) \rightsquigarrow \cdots \leftrightarrow, y\left(k_{1}, k_{2}, \ldots, k_{d}\right) .
\end{aligned}
$$

The $\leadsto$ represents a path in $G$ that follows the sub-cubes of a $P(i, j)$, choosing one vertex from each $K_{a}$-cube as necessary.

Thus we first increase the first component $\left(\bmod 1 / h_{b}\right)$ until it is $k_{1}$ and then do the same for the second and subsequent components. Each such path has length at most $3 d L_{1} / h_{b}=$ $O(1 / r)$. If we fix a grid edge $e$ (really an edge of a path $\rightsquigarrow$ ) joining $y\left(j_{1}, \ldots, j_{t}, \ldots, j_{d}\right)$ to $y\left(j_{1}, \ldots, j_{t}+1, \ldots, j_{d}\right)$ then the number of paths through $e$ is $O\left(h_{b}^{-d-1}\right)$; any such path starts at $y\left(l_{1}, \ldots, l_{t}, j_{t+1}, \ldots, j_{d}\right)$ and ends at $y\left(j_{1}, \ldots, j_{t-1}, l_{t}^{\prime}, \ldots, l_{d}^{\prime}\right)$ for some $l_{1}, \ldots, l_{t}, l_{t}^{\prime}, \ldots, l_{d}$.

We obtain canonical paths for every pair of vertices by using Lemma 10 i.e. we connect each point $x$ of $V$ to its closest $x_{i}=\phi(x)$. Each $x_{i}$ is chosen by $O(\log n)$ points in this way. Using 
Chernoff bounds, we bound the number of points in $V$ at $G$-distance $O(1)$ (Lemma 10) from any $x_{i}$. Our path from $x$ to $y$ goes $x$ to $\phi(x)$ to $\phi(y)$ to $y$. After this we find that each path has length $O(1 / r)$ and each edge is in $\tilde{O}\left(1 / r^{d+1}\right)$ paths. It follows from (15) that

$$
\rho=\tilde{O}\left(n \cdot 1 \cdot r^{-d-1} \cdot n^{-2} \cdot r^{-1}\right)=\tilde{O}\left(() 1 /\left(n r^{d+2}\right)\right)=\tilde{O}\left(n^{2 / d}\right)
$$

and the lemma follows from (14).

Applying (13) we see that we can take

$$
T=\tilde{O}\left(n^{2 / d}\right)
$$

when we use Lemma 11.

We note that Boyd, Ghosh, Prabhakar and Shah [6] and Bar-Yossef, Friedman and Kliot [5] have proved tight results on the mixing time for $p \geq C \log n / n$ and $C$ sufficiently large.

\section{Expected number of returns during the mixing time etc.}

Having obtained a good enough bound on $T$, we next show that whp $R_{v}=1+o(1)$ for all $v \in V$, and that the conditions of Lemma 11 hold.

For a set of vertices $B$, the escape probability $p_{\mathrm{esc}}(a, B)$, is the probability that a random walk leaving $a$ does not return to $a$ before reaching $B$. This probability is given by

$$
p_{\mathrm{esc}}(a, B)=\frac{1}{d(a) \mathrm{R}_{\mathrm{EFF}}(v, B)},
$$

where $\mathrm{R}_{\mathrm{EFF}}(a, B)$ is the effective resistance between $a$ and $B$ in an electrical network with all edges having resistance one, see for example Doyle and Snell [13].

Thus $R_{v}(B)$, the expected number of returns to $v$ before reaching $B$ is given by

$$
R_{v}(B)=\frac{1}{p_{\mathrm{esc}}(v, B)}=d(v) \mathrm{R}_{\mathrm{EFF}}(v, B) .
$$

Raleigh's Theorem (see e.g. [13]), states that deleting edges increases effective resistance. Thus, provided we do not prune edges incident with vertex $v$, edge deletion increases $R_{v}(B)$.

We will identify a set $U_{v}$ such that $\mathcal{W}_{v}$ is very likely to enter $U_{v}$ before returning to $v$ and such that if $x \in U_{v}$ then $\mathcal{W}_{x}$ is unlikely to visit $v$ within time $T$. We deduce from this the $R_{v}=1+o(1)$. 


\subsection{The probability a walk visits fixed vertex during $T$}

For $x, y \in V$ we let

$$
\eta(x, y)=\operatorname{Pr}\left(\exists 1 \leq t \leq T: \mathcal{W}_{x}(t)=y\right) .
$$

We aim to show that if $y$ is fixed, then $\eta(x, y)=o(1)$ for almost all choices of $x$.

Given $\epsilon>0$, let

$$
B_{\epsilon}(x)=\{y \in V: \eta(y, x) \geq \epsilon\} .
$$

By stationarity, for fixed $t$,

$$
\sum_{y \in V} \pi_{y} \operatorname{Pr}\left(\mathcal{W}_{y}(t)=x\right)=\pi_{x} .
$$

Thus

$$
\begin{aligned}
T \pi_{x} & =\sum_{1 \leq t \leq T} \sum_{y \in V} \pi_{y} P_{y}^{(t)}(x) \\
& =\sum_{y \in V} \pi_{y} \sum_{1 \leq t \leq T} P_{y}^{(t)}(x) \\
& \geq \sum_{y \in V} \pi_{y} \eta(y, x) \\
& \geq \sum_{y \in B_{x}(\epsilon)} \pi_{y} \eta(y, x) \\
& \geq \pi_{\min } \epsilon\left|B_{x}(\epsilon)\right| .
\end{aligned}
$$

where $\pi_{\min }=\min \left\{\pi_{y}: y \in V\right\}$.

Consequently,

$$
\left|B_{\epsilon}(x)\right| \leq \frac{T \pi_{x}}{\epsilon \pi_{\min }} .
$$

It follows that if

$$
U_{v}=\left\{x: \eta(x, v) \geq \frac{1}{(\log n)^{2}}\right\}
$$

then whp

$$
\left|U_{v}\right|=\tilde{O}(T) .
$$

\subsection{The value of $R_{v}$}

We next prove that

Lemma 13. Whp $R_{v}=1+O(1 / \log n)$ for all $v \in V$. 
Proof Fix $v \in V$ and make $v$ the centre of a $K_{b}$-cube $C_{v}$ of side $L_{1} h_{a}$ and partition $I(d)$ into $K_{b}$-cubes with $C_{v}$ as one of the cubes. Let $\bar{U}_{v}=V \backslash U_{v}$.

Let $\mathcal{W}_{v}$ starting from $v$, and let $p_{v}$ be the probability of a first return to $v$ within time $T$ by this walk. Then

$$
1 \leq R_{v} \leq \frac{1}{1-p_{v}},
$$

and

$$
p_{v} \leq 1-p_{\text {esc }}\left(v, \bar{U}_{v}\right)+1 /(\log n)^{2} .
$$

Given (21) and (20), it is sufficient to prove for all $v \in V$ that whp

$$
p_{\text {esc }}\left(v, \bar{U}_{v}\right)=1-O(1 / \log n) .
$$

We focus on proving (22). We construct a sub-graph $G_{v}^{*}=\left(V^{*}, E^{*}\right)$ of $G$. The set $V^{*}=$ $W_{1}^{*} \cup W_{2}^{*}$, where $W_{1}^{*}, W_{2}^{*}$ are defined as follows. Let $S$ denote the set of heavy $K_{a}$-cubes that either (i) belong to a path $P(i, j)$ as defined in Section 2 or (ii) are contained in $C_{v}$. For each sub-cube in $S$, we choose an arbitrary subset of vertices of size $\epsilon_{\ell} \alpha \log n, \alpha=c /\left(\Upsilon_{d} \epsilon_{a}^{d}\right)$ and place these vertices in $W_{1}^{*}$. (See the definition of heavy above Lemma 5 for the values of these constants.) Let $W_{2}^{*}$ consist of $v$ and all of its neighbours and any vertex that is in a heavy cube of $C_{v}$. Note that (3) implies that $v$ has no neighbours outside of $C_{v}$. Let $E^{*}=E_{1}^{*} \cup E_{2}^{*}$, where $E_{1}^{*}$ consists of those edges $(x, y)$ where $x, y$ are either contained in the same $K_{a}$-cube of $S$, or are in distinct $K_{a}$-cubes of $S$ which share a $(d-1)$-dimensional face. The set $E_{2}^{*}$ consists of the edges of $G$ that join two vertices of $W_{2}^{*}$.

The degree of $v$ in $G^{*}$ is the same as its degree in $G$ and $G^{*}$ is a sub-graph of $G$. From Raleigh's Theorem, we see that $\mathrm{R}_{\mathrm{EFF}}\left(v, \bar{U}_{v}\right) \leq \mathrm{R}_{\mathrm{EFF}}^{*}\left(v, \bar{U}_{v}\right)$. So if $p_{\mathrm{esc}}^{*}\left(v, \bar{U}_{v}\right)$ is the probability that the random walk $\mathcal{W}_{v}^{*}$ on $G^{*}$ visits $\bar{U}_{v}$ before returning to $v$ then

$$
p_{\text {esc }}\left(v, \bar{U}_{v}\right) \geq p_{\text {esc }}^{*}\left(v, \bar{U}_{v}\right) .
$$

So to prove (22), it suffices to prove

$$
p_{\text {esc }}^{*}\left(v, \bar{U}_{v}\right)=1-O(1 / \log n)
$$

Define a $d$-dimensional grid $\tilde{\Gamma}$ with $M$ vertices as follows: There is one vertex of $\tilde{\Gamma}$ for each of the $K_{b}$-cubes $B_{1}, B_{2}, \ldots, B_{M}$ in the partition of $I(d)$ and the cube $C_{v}$ corresponds to the origin of $\tilde{\Gamma}$.

With one small caveat, the random walk $\mathcal{W}_{v}^{*}$ on $G^{*}$ can be coupled with a random walk $\tilde{\mathcal{W}}$ on $\tilde{\Gamma}$. Let $V_{i}^{*}=V^{*} \cap A_{i}$, for $i=1,2, \ldots, M$, where $A_{i}$ is the useable $K_{a}$-cube of $B_{i}$ chosen according to Lemma 9 . When $\mathcal{W}_{v}^{*}$ is inside $V_{i}^{*}, \tilde{\mathcal{W}}$ will be at the $i$ th vertex of $\tilde{\Gamma}$. If $\mathcal{W}_{v}^{*}$ is on a vertex of a path $P(i, j)$ then $\tilde{\Gamma}$ stays at its current vertex. Since the paths $P(i, j)$ are all of the same length, and since the $V_{i}^{*}$ are all the same size, the next vertex that $\mathcal{W}_{v}^{*}$ visits 
is equally likely to be any neighbour of the current vertex. The caveat concerns what to do when $\mathcal{W}_{v}^{*}$ is inside $C_{v}$ or equivalently when $\tilde{\mathcal{W}}$ is at the origin. In this case it is not true that the $K_{b}$-cube next visited by $\mathcal{W}_{v}^{*}$ is equally likely to be any neighbour. This turns out to be unimportant, but it should be borne in mind in the following discussion.

Now focus on the random walk $\tilde{\mathcal{W}}$ on the $[N]^{d}, N=M^{1 / d}$, where $M=\Omega(n / \log n)$. By assumption, $\tilde{\mathcal{W}}$ starts at the origin. Let $\mathcal{J}_{V}^{*}=\left\{i \in[M]: V_{i}^{*} \subseteq \bar{U}_{v}\right\}$.

We will prove that with probability bounded below by a constant $\gamma>0, \tilde{\mathcal{W}}$ will (for some small constant $c_{1}$ ) visit $\mathcal{J}_{V}^{*}$ within $c_{1} N^{2}$, steps, before returning to the origin. We will also show that

$$
\operatorname{Pr}\left(\mathcal{W}_{v}^{*} \text { revisits } v \mid \mathcal{W}_{v}^{*} \text { visits } W_{2}^{*}\right) \leq \epsilon_{v}=O(1 / \log n) .
$$

This bound includes the probability of a re-visit to $v$ before $\mathcal{W}^{*}$ first leaves $C_{v}$. Thus,

$$
1-p_{\mathrm{esc}}^{*}\left(v, \bar{U}_{v}\right) \leq \epsilon_{v} \sum_{i=0}^{\infty} i(1-\gamma)^{i}<\frac{\epsilon_{v}}{\gamma^{2}}=O(1 / \log n)
$$

and this completes the proof of (23).

Now because $d \geq 3$ there is a positive probability $\gamma^{\prime}$ such that we have $\tilde{\mathcal{W}}\left(t^{\prime}\right) \neq 0$ for $1 \leq$ $t^{\prime} \leq t=c_{1} N^{2}$. This is because a random walk $\hat{\mathcal{W}}$ on the infinite $d$-dimensional lattice is nonrecurrent. Thus there is a constant probability $\zeta_{d}>0$ that it does not return to the origin. The symmetry of the grid ensures that this remains true, even if there is a non-uniform choice of neighbour at the origin. If $c_{1}$ is small, then there is a greater than $1-\zeta_{d} / 2$ chance that $\hat{\mathcal{W}}$ stays inside the box $[-N / 3, N / 3]^{d}$ for the first $t$ steps and this implies that $\tilde{\mathcal{W}}$ does not return to the origin with probability at least $\zeta_{d}-\zeta_{d} / 2$. Furthermore, if the walk does not return to the origin, then its subsequent behaviour is precisely that of the standard walk, given the neighbour $X_{1}=\tilde{\mathcal{W}}(1)$ of the origin that is first chosen. So we can now think of the distribution of $\tilde{\mathcal{W}}$ as that of a standard walk that first goes to $X_{1}$ and then with probability at least $\zeta_{d} / 2$ does not return to the origin. Now for a fixed $x \in N^{d}$ we have

$$
\operatorname{Pr}\left(\mathcal{W}_{v}^{*}(t) \in V_{x}^{*} \mid \text { no return, } X_{1}\right)=O\left(\operatorname{Pr}\left(\mathcal{W}_{v}^{*}(t) \in V_{x}^{*} \mid X_{1}\right)\right)=O\left(\operatorname{Pr}\left(\hat{\mathcal{W}}(t)=x \mid X_{1}\right)\right.
$$

For a random walk $\hat{\mathcal{W}}$ on the $d$-dimensional lattice,

$$
\operatorname{Pr}(\hat{\mathcal{W}}(t)=x)=O(\operatorname{Pr}(\hat{\mathcal{W}}(t)=0))=O\left(t^{-d / 2}\right)
$$

This can be seen by considering the relevant multinomial terms (see e.g. page 329 of Feller [17] for the case $d=3$ ). Thus choosing $t=c_{1} N^{2}$, as above, we have

$$
\operatorname{Pr}\left(\tilde{\mathcal{W}}_{v}(t) \notin \mathcal{J}_{V}^{*}\right)=\tilde{O}\left(\left|U_{v}\right| N^{-d}\right)=\tilde{O}\left(T N^{-d}\right)=O(1 / \log n) .
$$

So

$$
\operatorname{Pr}\left(\mathcal{W}_{v}^{*}(t) \notin \bigcup_{i \in \mathcal{J}_{V}^{*}} V_{i}^{*}\right)=\tilde{O}\left(\left|U_{v}\right| N^{-d}\right)=\tilde{O}\left(T N^{-d}\right)=O(1 / \log n) .
$$

Now any constant $\gamma<\gamma^{\prime}$ will suffice. 


\subsection{Proof of (24):}

For this we consider the graph $H$ with vertex set equal to the set of heavy $K_{a}$-cubes. Two heavy cubes $C_{1}, C_{2}$ are defined to be adjacent in $H$ if the centers of $C_{1}, C_{2}$ are no more than $r_{1}=r-2 d^{1 / 2} h_{a}$ apart. In which case, $v_{i} \in C_{i} \cap V, i=1,2$ implies that $\left(v_{1}, v_{2}\right) \in G$.

Lemma 14. The ball $D(v, r)$ contains $\Upsilon_{d} \epsilon_{a}^{-d}\left(1-\epsilon_{B}\right) K_{a}$-cubes, where $0 \leq \epsilon_{B} \leq 1-(1-$ $\left.2 \epsilon_{a} d^{1 / 2}\right)^{d}$.

Proof The upper bound of $\frac{\Upsilon_{d} r^{d}}{\left(\epsilon_{a} r\right)^{d}}$ is the ratio of the volume of $D(v, r)$ and the volume of a $K_{a}$-cube. For the lower bound, consider $D\left(v, r^{\prime}\right), r^{\prime}=r_{1}$. Every $K_{a}$-cube that touches $D\left(v, r_{1}\right)$ is contained entirely in $D(v, r)$. There are at least $\frac{\Upsilon_{d} r_{1}^{d}}{\left(\epsilon_{a} r\right)^{d}}$ cubes that touch $D\left(v, r_{1}\right)$ and the lower bound follows.

It follows that the maximum degree in $H$ satisfies

$$
\Delta(H) \leq \Upsilon_{d} \epsilon_{a}^{-d}=O(1)
$$

Lemma 15. Whp, for every $w \in V, D(w, r)$ contains at least one heavy cube.

Proof Let $c^{\prime}=\Upsilon_{d} r_{1}^{d} n / \log n=c\left(1-2 d^{1 / 2} \epsilon_{a}\right)^{d}$. If $\epsilon_{a}$ is sufficiently small, we will have $c^{\prime}>1$ and then whp (see proof of Lemma 3) $D\left(w, r_{1}\right)$ contains at least $c_{0}^{\prime} \log n$ points of $V$ where $c_{0}^{\prime} \geq c_{0} / 2$, for all $w \in V$. There are at most $\Upsilon_{d} \epsilon_{a}^{-d}$ cubes contained entirely in $D(w, r)$. So, one of these cubes must contain at least $\frac{c_{0} \epsilon_{a}^{d}}{2 \Upsilon_{d}} \log n$ points i.e. is heavy.

The following bound is somewhat crude, but it will suffice.

Lemma 16. Whp $H$ contains no component with fewer than $\log \log n$ vertices.

Proof Let $\delta_{C}=1_{C}$ is heavy for a $K_{a}$-cube $C$. Now let $C$ be a fixed $K_{a}$-cube and $S$ a set of $K_{a}$-cubes with $|S|=O(\log n)$. If $p=\frac{c \epsilon_{a}^{d} \log n}{\Upsilon_{d} n}=\frac{\alpha \log n}{n}$, and $n^{\prime}=n-O\left((\log n)^{2}\right)$ then

$$
\operatorname{Pr}\left(C \text { is light } \mid \delta_{s}, s \in S\right) \leq \sum_{i=0}^{\epsilon_{\ell} \alpha \log n}\left(\begin{array}{c}
n^{\prime} \\
i
\end{array}\right) p^{i}(1-p)^{n^{\prime}-i} \leq n^{-\beta}
$$

where $\beta=\alpha\left(1-\epsilon_{\ell} \log \left(e / \epsilon_{\ell}\right)\right)+o(1)$. (Strictly speaking we should also condition on the event that every $K_{a}$-cube has $O(\log n)$ points of $V$. However, this happens with probability $1-O\left(n^{-K}\right)$ where the constant $K$ can be made as large as necessary).

Let $N=1 / \epsilon_{a} r$ so that the grid $K_{a}$ has $N^{d}$ cubes.

Case 1: $k \leq(c-1) \Upsilon_{d} \epsilon_{a}^{-d} / 2$. 
The probability that $H$ contains a component with $k$ vertices is at most

$$
o(1)+n\left(e \epsilon_{a}^{-d}\right)^{k} n^{-\beta\left(\Upsilon_{d} \epsilon_{a}^{-d}\left(1-\epsilon_{B}\right)\left(r_{1} / r\right)^{d}-k\right)}=o(1) .
$$

Explanation: The $o(1)$ term on the LHS is the probability that there is a vertex of degree exceeding $(c+10) \log n$. Choose a $K_{a}$-cube $C$ and another $k-1$ that make up a connected component of $H$. There are at most $\left(e \epsilon_{a}^{-d}\right)^{k}$ ways to choose a tree of cubes with root $C$. Now let $x$ be the centre of the first chosen cube. The term $n^{-\beta\left(\Upsilon_{d} \epsilon_{a}^{-d}\left(1-\epsilon_{B}\right)\left(r_{1} / r\right)^{d}-k\right)}$ bounds the probability that all of the other cubes in $D\left(x, r_{1}\right)$ are light. Note that $\beta\left(\Upsilon_{d} \epsilon_{a}^{-d}\left(1-\epsilon_{B}\right)\left(r_{1} / r\right)^{d}-\right.$ $k)>1$ if $\epsilon_{\ell}, \epsilon_{B}=\epsilon_{B}\left(\epsilon_{a}\right)$ are sufficiently small.

Case 2: $(c-1) \Upsilon_{d} \epsilon_{a}^{-d} / 2 \leq k \leq \log \log n$.

Choose $k K_{a}$-cubes $C_{1}, C_{2}, \ldots, C_{k}$ and let $X=\bigcup_{i=1}^{k} C_{i}$. Let $Y$ be the subset of $I(d)$ within distance $r_{2}=r_{1}-d^{1 / 2} h_{a}$ of $X$. Note that if $y \in Y$ then it is within distance $r_{1}$ of a centre of a sub-cube of $X$. We claim that

$$
\operatorname{vol}_{d}(Y) \geq \Upsilon_{d}\left(\rho+r_{2}\right)^{d}
$$

where $\rho$ is the solution to $k \epsilon_{a}^{d} r^{d}=\Upsilon_{d} \rho^{d}$. This follows from the classical isoperimetric inequality [4], where $\rho$ is the radius of a ball with the same volume as $X$. Note that

$$
\rho=\left(\frac{k \epsilon_{a}^{d} r^{d}}{\Upsilon_{d}}\right)^{1 / d}=\left(\frac{k}{\Upsilon_{d}}\right)^{1 / d} \epsilon_{a} r \geq\left(\frac{c-1}{2}\right)^{1 / d} r
$$

Arguing as in Lemma 14 we see that if $r_{3}=r_{2}-2 d^{1 / 2} h_{a}$ then $Y \backslash X$ contains at least

$$
\Upsilon_{d} \epsilon_{a}^{-d}\left(\left(1-\epsilon_{B}\right)\left(\frac{\rho+r_{3}}{r}\right)^{d}-\left(\frac{\rho}{r}\right)^{d}\right) \geq \Upsilon_{d} \epsilon_{a}^{-d}\left(1-\epsilon_{B}\right)\left(r_{3} / r\right)^{d}
$$

$K_{a}$-cubes. So, the probability that $H$ contains a component with $k$ vertices is at most

$$
n((c+10) \log n)^{k} n^{-\beta\left(\Upsilon_{d} \epsilon_{a}^{-d}\left(1-\epsilon_{B}\right)\left(r_{3} / r\right)^{d}\right)}=o(1) .
$$

The term $n^{-\beta\left(\Upsilon_{d} \epsilon_{a}^{-d}\left(1-\epsilon_{B}\right)\left(r_{3} / r\right)^{d}\right)}$ bounds the probability that all the cubes mentioned in (27) are light.

Now consider a random walk $\mathcal{W}^{*}$ on $G^{*}$ that starts at some vertex $w \in C_{v}$. Note first that, when at a neighbour of $v$, there is only an $O(1 / \log n)$ chance of returning to $v$ at the next step. Second note, that at any vertex, there is at least the chance $\epsilon_{h}=\frac{\epsilon_{\ell} \alpha}{c+10}$ of moving to a heavy cube (Lemma 15). In particular, with probability at least $\epsilon_{h}-o(1)$ the next vertex after $w$ lies in a heavy cube and is not equal to $v$. Let $P$ be some path in $G^{*}$ of length $t_{0}$ where $20 \Upsilon_{d} \epsilon_{a}^{-d} \ll t_{0} \ll L_{1}$. We can use Lemma 16 to argue that $P$ exists. 
Then note that with probability $\left(\frac{\epsilon_{h}}{\Delta(H)}\right)^{t_{0}}$ the next $t_{0}$ steps of $\mathcal{W}^{*}$ traverse the cubes of $P$. By Lemma 5 we see that at least one of the cubes on $P$ will be in $\kappa_{1}\left(C_{v}\right)$. Once we reach such cube there is at least the chance $\epsilon^{*}=\epsilon_{h}^{3^{d}\left(\Gamma_{d}+c_{b} \Gamma_{d}^{2}\right)+L_{1}^{d}+1}$ of leaving $C_{v}$ by going along the path promised by Lemma 10 to a giant component and then going through this giant component and leaving $C_{v}$. Thus the chance of returning to $v$ is $O(1 / \log n)$ either when starting at $v$ or when returning to $C_{v}$.

This completes the proof of (24) and the lemma.

\subsection{Conditions of Lemma 11}

It is clear from (16) that Lemma 11(b) holds. To check condition (a) we see that if $|z| \leq 1+\lambda$ then since by Lemma 13 we have $R_{v}=1+o(1)$, we see, using (11) that

$$
\left|\sum_{j=1}^{T-1} r_{j} z^{j}\right| \leq(1+\lambda)^{T} \sum_{j=1}^{T-1} r_{t}=(1+\lambda)^{T}\left(R_{v}-1\right) \leq e^{1 / K}\left(R_{v}-1\right)=o(1) .
$$

So $\left|R_{T}(z)\right| \geq 1-\left|\sum_{j=1}^{T-1} r_{j} z^{j}\right|=1-o(1)$ for $|z| \leq 1+\lambda$.

\section{Cover time}

From (12) of Lemma 11 we have that for all $t \geq T$,

$$
\operatorname{Pr}\left(\mathcal{A}_{t}(v)\right)=\frac{1+o(1)}{\left(1+\frac{\pi_{v}}{R_{v}\left(1+O\left(T \pi_{v}\right)\right)}\right)^{t+1}}+O\left(T e^{-\lambda t / 2}\right)
$$

An upper bound is obtained as follows: Let $T_{G}(u)$ be the time taken to visit every vertex of $G$ by the random walk $\mathcal{W}_{u}$. Let $U_{t}$ be the number of vertices of $G$ which have not been visited by $\mathcal{W}_{u}$ at step $t$. We note the following:

$$
\begin{aligned}
\operatorname{Pr}\left(T_{G}(u)>t\right) & =\operatorname{Pr}\left(U_{t}>0\right) \leq \min \left\{1, \mathbf{E} U_{t}\right\} \\
C_{u}=\mathbf{E} T_{G}(u) & =\sum_{t>0} \operatorname{Pr}\left(T_{G}(u)>t\right)
\end{aligned}
$$

It follows from $(29,30)$ that for all $t$

$$
C_{u} \leq t+1+\sum_{s>t} \mathbf{E} U_{s} \leq t+1+\sum_{v \in V} \sum_{s>t} \operatorname{Pr}\left(\mathcal{A}_{s}(v)\right) .
$$


Let

$$
t^{*}=\left(c \log \left(\frac{c}{c-1}\right)\right) n \log n
$$

and

$$
t_{0}=(1-\delta) t^{*} \text { and } t_{1}=(1+\delta) t^{*}
$$

where $\delta=o(1)$ but goes to zero sufficiently slowly that inequalities below are satisfied.

\subsection{Upper bound on the cover time}

For $v \in V$ we have by Lemmas 11 and 13 that for $s \geq T$

$$
\operatorname{Pr}\left(\mathcal{A}_{s}(v)\right)=(1+o(1)) \exp \left\{-(1+o(1 / \log n)) \pi_{v} s\right\}+O\left(T e^{-\Omega(s / T)}\right)
$$

and we note that

$$
\pi_{v}=\frac{d(v)}{2 m} .
$$

Then we find, using the whp bounds in Lemma 4,

$$
C_{u} \leq t_{1}+1+S_{1}+S_{2}+O\left(n T^{2} e^{-\Omega(s / T)}\right)
$$

where

$$
\begin{aligned}
S_{i} & =\sum_{k \in K_{i}} D(k) \sum_{s \geq t_{1}} \exp \left\{-\frac{(1-o(1)) k s}{2 m}\right\} \\
& \leq 3 m \sum_{k \in K_{i}} \frac{D(k)}{k} e^{-(1-o(1)) k t_{1} / 2 m} \\
& \leq 3 m \sum_{k \in K_{i}} \frac{D(k)}{k}\left(\frac{c-1}{c}\right)^{(1+\delta / 2) k} .
\end{aligned}
$$

For the first term,

$$
\begin{aligned}
S_{1} & \leq 3 m \sum_{k \in K_{1}} \frac{(\log n)^{4}}{k}\left(\frac{c-1}{c}\right)^{(1+\delta / 2) k} \\
& =o\left(t_{1}\right)
\end{aligned}
$$

since $D(k) \leq(\log n)^{4}$ and $\min \left\{k \in K_{2}\right\} \geq(\log n)^{1 / 2}$. 
Continuing we get

$$
\begin{aligned}
S_{2} & \leq \frac{6 m}{n^{c-1}} \sum_{k \in I_{c}}\left(\frac{n e p}{k}\right)^{k}\left(\frac{c-1}{c}\right)^{(1+\delta / 2) k} \\
& \leq 6 m \sum_{k \in I_{c}} e^{-\delta k / 2 c} \\
& =o\left(t_{1}\right),
\end{aligned}
$$

where we have used the fact that $(n e p(c-1)) /(k c))^{k}$ is maximized at $k=n p(c-1) / c$, and $\delta k / \log \log n \rightarrow \infty$ for $k \in I_{c}$.

It now follows from (32) - (33) that $C_{u} \leq t_{1}+o\left(t_{1}\right)$.

\subsection{Lower bound on the cover time}

We can find a vertex $u$ and a set of vertices $S_{0}$ such that at time $t_{0}$, the probability the set $S_{0}$ is covered by the walk $\mathcal{W}_{u}$ tends to zero. Hence $T_{G}(u)>t_{0}$ whp which implies that $C_{G} \geq(1-o(1)) t^{*}$.

We construct $S_{0}$ as follows. Let $k_{1}=(c-1) \log n$ be as defined in Lemma 4, and let $S_{1}=\left\{v: d(v)=k_{1}\right\}$. Let $A=\left\{(u, v): u \notin S_{1}, v \in S_{1}, \eta(u, v) \geq 1 /(\log n)^{2}\right\}$, where $\eta(u, v)$ is defined in (18). It follows from (19) that whp $|A|=\tilde{O}\left(T\left|S_{1}\right|\right)$. By simple counting, we see that there exists $u \notin S_{1}$ such that $\left|\left\{v \in S_{1}:(u, v) \in A\right\}\right|=\tilde{O}\left(T\left|S_{1}\right| / n\right)=o\left(\left|S_{1}\right|\right)$. We choose such a $u$ and let $S_{2}=\left\{v \in S_{1}:(u, v) \notin A\right\}$. We then take an independent subset $S_{0}$ of $S_{2}$. Because the maximum degree of $G$ is $O(\log n)$ we can choose $\left|S_{0}\right|=\Omega\left(\left|S_{2}\right| / \log n\right)$.

Let $\mathcal{B}_{v}$ be the event that $\mathcal{W}_{u}$ does not visit $v$ in the time interval $[1, T]$. Then, by our choice of $u$, we see that for $v \in S_{0}$,

$$
\operatorname{Pr}\left(\mathcal{B}_{v}\right) \geq 1-1 /(\log n)^{2} .
$$

We need to prove that

$$
\operatorname{Pr}\left(\mathcal{A}_{t_{0}}(v) \mid \mathcal{B}_{v}\right)=(1+o(1)) \exp \left\{-\frac{(c-1) t_{0}(1+o(1))}{c n R_{v}\left(1+O\left(T \pi_{v}\right)\right)}\right\}=(1+o(1)) \operatorname{Pr}\left(\mathcal{A}_{t_{0}}(v)\right) .
$$

The proof of this requires just a small change to the proof of Lemma 11, which we include as an appendix. (Equality is needed in (36). The reader will observe that $\operatorname{Pr}\left(\mathcal{A}_{t}(v) \mid \mathcal{B}_{v}\right) \leq$ $(1+o(1)) \operatorname{Pr}\left(\mathcal{A}_{t}(v)\right)$ follows immediately from (35)).

Then whp, if $Z_{0}$ is the number of vertices in $S_{0}$ that are not visited in time $\left[1, t_{0}\right]$,

$$
\mathbf{E}\left(Z_{0}\right) \geq A_{1} \frac{n^{(c-1) \log (c /(c-1))}}{\log n} \exp \left\{-(1+o(1)) \frac{(c-1) t_{0}}{c n}\right\} \geq A_{2} n^{\frac{1}{2} \delta(c-1) \log (c /(c-1))} \rightarrow \infty
$$


for some constants $A_{1}, A_{2}>0$.

We show next, for all $v, w \in S_{0}$, that

$$
\eta(v, w)=O(1 / \log n) .
$$

We define a new graph $G_{\psi}$ by identifying $v, w$ and replacing them with a new node $\psi$. The proof of Lemma 12 can be modified to show that the mixing time $T_{\psi}$ of $G_{\psi}$ will satisfy (16). Indeed, we can assume that our choice of $x_{i}$ 's excludes $v, w$ and then $v, w$ can only appear as endpoints of canonical paths. For a path from $x$ to $\psi$ we can then choose one of the already constructed canonical paths from $x$ to $v$ or $x$ to $w$.

Similarly, the proof of Lemma 13 can be modified to show that

$$
p_{\text {esc }}\left(\psi, \bar{U}_{\psi}\right)=1-O(1 / \log n) .
$$

Here $\bar{U}_{\psi}=\bar{U}_{v} \cap \bar{U}_{w}$ and the probability is for a random walk in $G_{\psi}$ starting at $\psi$. Our modification of Lemma 13 requires a random walk on the $d$-dimensional lattice, starting at point $x$ (a surrogate for $v$ 's cube), to have positive probability of not returning to $x$ or some other fixed vertex $y$ (a surrogate for w's cube) and vice-versa. This is a simple consequence of Pólya's classic result.

Then

$$
\eta(v, w) \leq 2\left(1-p_{\text {esc }}\left(\psi, U_{\psi}\right)\right)+O\left(1 /(\log n)^{2}\right)=O(1 / \log n) .
$$

The term $2\left(1-p_{\text {esc }}\left(\psi, U_{\psi}\right)\right)$ arises as follows: Consider the random walk $\mathcal{W}_{\psi}$, conditioned on moving to a neighbour of $v$ at the first step. Up until the time that this walk returns to $\psi$ it behaves just like $\mathcal{W}_{v}$. It has a probability $1-p_{\text {esc }}\left(\psi, U_{\psi}\right)$ of not entering $U_{\psi}$ before returning to $\psi$ and this can be inflated by a factor 2 to account for the initial conditioning. But then $\mathcal{W}_{v}$ has a probability of at most $2\left(1-p_{\text {esc }}\left(\psi, U_{\psi}\right)\right)$ of avoiding $U_{\psi}$ before reaching $v$ or $w$ and a fortiori it has a probability of at most $2\left(1-p_{\text {esc }}\left(\psi, U_{\psi}\right)\right)$ of avoiding $U_{\psi}$ before reaching $w$. The term $O\left(1 /(\log n)^{2}\right)$ accounts for walks that reach $U_{\psi}$ before $w$.

Now for $v, w \neq u$ let

$$
\mathcal{A}_{t}(v, w)=\mathcal{A}_{t}(v) \wedge \mathcal{A}_{t}(w) \text { and } \mathcal{B}_{v, w}=\mathcal{B}_{v} \wedge \mathcal{B}_{w} .
$$

Then for $v \neq w$,

$$
\operatorname{Pr}\left(\mathcal{B}_{v, w}\right) \geq 1-\operatorname{Pr}\left(\overline{\mathcal{B}}_{v}\right)-\operatorname{Pr}\left(\overline{\mathcal{B}}_{w}\right) \geq 1-2 /(\log n)^{2}
$$

and we will show that

$$
\operatorname{Pr}\left(\mathcal{A}_{t_{0}}(v, w) \mid \mathcal{B}_{v, w}\right) \leq A_{0} \operatorname{Pr}\left(\mathcal{A}_{t_{0}}(v)\right) \operatorname{Pr}\left(\mathcal{A}_{t_{0}}(w)\right) .
$$

for all $v, w \in S_{0}$, for some absolute constant $A_{0}$ and

$$
\operatorname{Pr}\left(\mathcal{A}_{t_{0}}(v, w) \mid \mathcal{B}_{v, w}\right)=(1+o(1)) \operatorname{Pr}\left(\mathcal{A}_{t_{0}}(v, w)\right)=(1+o(1)) \operatorname{Pr}\left(\mathcal{A}_{t_{0}}(v)\right) \operatorname{Pr}\left(\mathcal{A}_{t_{0}}(w)\right)
$$


for almost all pairs $(v, w) \in S_{0}$.

It then follows that

$$
\mathbf{E}\left(Z_{0}\left(Z_{0}-1\right)\right) \leq(1+o(1)) \mathbf{E}\left(Z_{0}\right)^{2}
$$

and so

$$
\operatorname{Pr}\left(Z_{0} \neq 0\right) \geq \frac{\mathbf{E}\left(Z_{0}\right)^{2}}{\mathbf{E}\left(Z_{0}^{2}\right)}=\frac{1}{\frac{\mathbf{E}\left(Z_{0}\left(Z_{0}-1\right)\right)}{\mathbf{E}\left(Z_{0}\right)^{2}}+\left(\mathbf{E} Z_{0}\right)^{-1}}=1-o(1)
$$

from (37) and (42).

\subsubsection{Proof of (41)}

We argue that

$$
R_{\psi} \leq \frac{R_{v}+R_{w}}{2}+O(1 / \log n)
$$

Walks in $G_{\psi}$ can be mapped to walks in $G$ in a natural way. If the walk is not at $\psi$ then it chooses its successor with the same probability. When at $\psi$, with probability $1 / 2$ it moves to a neighbour of $v$ and with probability $1 / 2$ it moves to a neighbour of $w$. Returns to $v, w$ account for the term $\frac{R_{v}+R_{w}}{2}$. We must also account for returns to $\psi$ that come from walks from $v$ to $w$ and vice-versa. This can be overestimated by $R_{v} \eta(v, w)+R_{w} \eta(w, v)$, giving the $O(1 / \log n)$ term.

Putting $\pi_{v}=\pi_{w}=\pi_{0}=\pi_{\psi} / 2$, this implies that

$$
\begin{aligned}
\frac{\pi_{\psi}}{R_{\psi}}-\frac{\pi_{v}}{R_{v}}-\frac{\pi_{w}}{R_{w}} & =\frac{\pi_{0}}{R_{\psi} R_{v} R_{w}}\left(2 R_{v} R_{w}-R_{\psi}\left(R_{v}+R_{w}\right)\right) \\
& \geq \frac{\pi_{0}}{R_{\psi} R_{v} R_{w}}\left(2 R_{v} R_{w}-\left(\frac{R_{v}+R_{w}}{2}+O(1 / \log n)\right)\left(R_{v}+R_{w}\right)\right) \\
& =\frac{\pi_{0}}{2 R_{\psi} R_{v} R_{w}}\left(\left(R_{v}-R_{w}\right)^{2}+O(1 / \log n)\right) \\
& =O\left(\frac{1}{n \log n}\right) .
\end{aligned}
$$

So, with $\mathbf{P r}_{\psi}$ referring to probability in the space of random walks on $G_{\psi}$,

$$
\begin{aligned}
\operatorname{Pr}_{\psi}\left(\mathcal{A}_{t_{0}}(\psi)\right) & =(1+o(1)) \exp \left\{-\frac{t_{0} \pi_{\psi}}{\left(1+O\left(T \pi_{\psi}\right)\right) R_{\psi}}\right\} \\
& =(1+o(1)) \exp \left\{-\frac{t_{0} \pi_{v}}{R_{v}}\right\} \exp \left\{-\frac{t_{0} \pi_{w}}{R_{w}}\right\} \exp \left\{O\left(\frac{t_{0}}{n \log n}\right)\right\} \\
& =O\left(\operatorname{Pr}\left(\mathcal{A}_{t_{0}}(v)\right) \operatorname{Pr}\left(\mathcal{A}_{t_{0}}(w)\right)\right) .
\end{aligned}
$$


But, using rapid mixing in $G_{\psi}$,

$$
\operatorname{Pr}_{\psi}\left(\mathcal{A}_{t_{0}}(\psi)\right)=\sum_{x \neq \psi} P_{\psi, u}^{T_{\psi}}(x) \operatorname{Pr}_{\psi}\left(\mathcal{W}_{x}\left(t-T_{\psi}\right) \neq \psi, T_{\psi} \leq t \leq t_{0}\right)
$$

The term $P_{\psi, u}^{T_{\psi}}(x)$ is the probability that a walk on $G_{\psi}$ that starts at $u$ will be at $x$ after $T_{\psi}$ steps.

$$
\begin{aligned}
& =\sum_{x \neq \psi}\left(\frac{d(x)}{2 m}+O\left(n^{-3}\right)\right) \operatorname{Pr}_{\psi}\left(\mathcal{W}_{x}\left(t-T_{\psi}\right) \neq \psi, T_{\psi} \leq t \leq t_{0}\right) \\
& =\sum_{x \neq v, w}\left(P_{u}^{T_{\psi}}(x)+O\left(n^{-3}\right)\right) \operatorname{Pr}\left(\mathcal{W}_{x}\left(t-T_{\psi}\right) \neq v, w, T_{\psi} \leq t \leq t_{0}\right) \\
& =\operatorname{Pr}\left(\mathcal{W}_{u}(t) \neq v, w, T_{\psi} \leq t \leq t_{0}\right)+O\left(n^{-2}\right) \\
& =\operatorname{Pr}\left(\mathcal{A}_{t_{0}}(v, w)\right)+O\left(n^{-2}\right) .
\end{aligned}
$$

Equation (46) follows because there is a natural measure preserving map $\phi$ between walks in $G$ that start at $x \neq v, w$ and avoid $v, w$ and walks in $G_{\psi}$ that avoid $\psi$. The map $\phi$ also shows that

$$
\operatorname{Pr}\left(\mathcal{A}_{t_{0}}(v, w) \wedge \mathcal{B}_{v, w}\right)=\operatorname{Pr}_{\psi}\left(\mathcal{A}_{t_{0}}(\psi) \wedge \mathcal{B}_{\psi}\right)=(1+o(1)) \operatorname{Pr}_{\psi}\left(\mathcal{A}_{t_{0}}(\psi) \mid \mathcal{B}_{\psi}\right)
$$

But the argument for (36) can be used to show that

$$
\operatorname{Pr}_{\psi}\left(\mathcal{A}_{t_{0}}(\psi) \mid \mathcal{B}_{\psi}\right)=(1+o(1)) \operatorname{Pr}_{\psi}\left(\mathcal{A}_{t_{0}}(\psi)\right) .
$$

Equation (41) follows from (45)-(49).

\subsection{Proof of (42)}

We get this sharpening of (41) whenever we can replace the $O(1 / \log n)$ in $(43)$ by $o(1 / \log n)$. This replacement can be done whenever we can replace $O(1 / \log n)$ in $(38)$ by $o(1 / \log n)$. We show that this can be done for almost all pairs $v, w \in S_{0}$.

There is a very simple argument when $c$ is sufficiently large. The size of $S_{0}$ is $n^{\gamma_{c}+o(1)} \mathbf{w h p}$ where $\gamma_{c}=(c-1) \log \left(\frac{c}{c-1}\right)$. For any fixed $v \in S_{0}$ there are at most $T(\log n)^{2}$ vertices $w$ such that $\eta(v, w) \geq 1 /(\log n)^{2}$. If $\gamma_{c}>2 / d$ then whp $T(\log n)^{2}=\tilde{O}\left(n^{2 / d}\right)=o\left(\left|S_{0}\right|\right)$ and (42) holds. For example, if $c \geq 2$ then $\gamma_{c} \geq \log 2=.69314718>2 / d$ for $d \geq 3$.

So now we must consider the case where $1<c \leq 2$. Let $A$ denote the set of unordered pairs $v, w \in S_{0}$ such that either $\eta(v, w) \geq 1 /(\log n)^{2}$ or $\eta(w, v) \geq 1 /(\log n)^{2}$. To prove (42) it is enough to show that

$$
\operatorname{Pr}\left(\eta(v, w) \geq 1 /(\log n)^{2}\left|v, w \in S_{0},\right| v-w \mid \geq r^{1 / 2}\right)=o(1) .
$$


Here, if $v=\left(v_{1}, v_{2}, \ldots, v_{d}\right)$ then $|v|=\left(v_{1}^{2}+v_{2}^{2}+\cdots+v_{d}^{2}\right)^{1 / 2}$.

Note that the expected number of pairs $v, w \in S_{0}$ such that $|v-w| \leq r^{1 / 2}$ can be bounded by $\tilde{O}\left(\max \left\{n^{2 \gamma_{c}-1 / 2+o(1)}, 1\right\}\right)$. So whp there are at most $\log n$ times this quantity. These pairs can therefore be ignored in our verification of (42).

To prove (50) we choose two points $v, w$ for which $|v-w| \geq r^{1 / 2}$, condition on $v, w \in S_{0}$ and then bound $\operatorname{Pr}(\eta(v, w))$ from below. We condition on $v, w \in S_{0}$ by randomly placing $k_{1}$ points into each of $D(v, r), D(w, r)$. We then couple part of the remaining construction of $G$ along with the first $T$ steps $\left(x_{1}, x_{2}, \ldots, x_{T}\right)$ of the random walk $\mathcal{W}_{v}$. We weaken $x_{T}=w$ to $x_{T} \in D(w, r)$.

Let $i_{v}=\max \left\{i: x_{i} \in D(v, 2 r)\right\}$. Notice next that for $i>i_{v}, x_{i+1}$ is either (i) chosen from some previously exposed point or (ii) randomly chosen from $D\left(x_{i}, r\right)$ and these latter choices are made independently. Let $J_{0}$ be the set of indices $i>i_{v}$ where a choice is made according to (ii). Suppose now that $i \in J_{0}$ and $x_{i}=\left(x_{i, 1}, x_{i, 2}, \ldots, x_{i, d}\right)$ and let $y_{i, j}=x_{i, j}-x_{i-1, j}$. The $y_{i, j}, j=1,2, \ldots, d$ are not independent. Their sum of squares is at most $r^{2}$. On the other hand, if $B\left(x_{i}\right)$ is the cube of side $2 r / d^{1 / 2}$ with centre $x_{i}$, then $B\left(x_{i}\right)$ is contained in $D\left(x_{i}, r\right)$. Conditional on $x_{i} \in B\left(x_{i-1}\right)$, the $y_{i, j}, j=1,2, \ldots, d$ are independent. Let $J_{1}$ be the indices $i \in J_{0}$ for which $x_{i} \in B\left(x_{i-1}\right)$. Then let $I_{t}=[t] \cap J_{1}$ for $t \geq 0$.

The size of $I_{t}$ is $\operatorname{Bin}\left(\left|J_{0} \cap[t]\right|, q\right)$ where $q$ is bounded away from 0. Furthermore, to reach $w$ in $t$ steps, we must have $\left|J_{0} \cap[t]\right| \geq|v-w| / r-O(\log n) \geq r^{-1 / 2}-O(\log n)$. So, by use of the Chernoff bounds, we can assume that $\left|I_{1}\right| \geq r^{-1 / 2} q / 2$. Now fix $I_{1}$ and condition on the values $y_{i, j}, i \notin I_{1}$ and let $Z_{j}=\sum_{i \in I_{1}} y_{i, j}, j=1,2, \ldots, d$. Now we have $Z_{1}, Z_{2}, \ldots, Z_{d}$ independent. Fix $j$. Then $Z_{j}=\sum_{l=1}^{s} \xi_{l}$ where whp $s \geq r^{-1 / 2} q / 2$ and $\xi_{l}$ is uniform in $\left[-r / d^{1 / 2}, r / d^{1 / 2}\right]$. As such it is well approximated by a normal distribution. In particular we can use the Berry-Esseen inequality, see for example [14]:

Let $X_{1}, X_{2}, \ldots, X_{N}$ be i.i.d. with $\mathbf{E}\left(X_{i}\right)=0, \mathbf{E}\left(X_{i}^{2}\right)=\sigma^{2}$ and $\mathbf{E}\left(\left|X_{i}\right|^{3}\right)=\rho<\infty$. If $F_{N}(x)$ is the distribution of $\left(X_{1}+X_{2}+\cdots+X_{N}\right) /(\sigma \sqrt{N})$ and $\mathcal{N}(x)$ is the standard normal distribution, then

$$
\left|F_{N}(x)-\mathcal{N}(x)\right| \leq \frac{3 \rho}{\sigma^{3} \sqrt{N}}
$$

To have $x_{t} \in D(w, r)$ each $Z_{j}$ will have to have to take a value in an interval $A_{j}$ of length at most $2 r$. This interval being determined by the values $x_{i}, i \notin I_{t}$. It follows from the BerryEsseen inequality that $\operatorname{Pr}\left(Z_{j} \in A_{j}\right)=O\left(t^{-1 / 2}\right)$. (We have $\sigma=\Omega(r)$ and $\rho=O\left(r^{3}\right)$ ). Hence, for some constant $C$,

$$
\mathbf{E}(\eta(v, w)) \leq C \sum_{t=r^{-1 / 2} q / 2}^{T} t^{-d / 2}=O\left(r^{1 / 4}\right)
$$

and (50) and (42) follow. 


\section{References}

[1] D. Aldous and J. Fill. Reversible Markov Chains and Random Walks on Graphs, http://stat-www.berkeley.edu/pub/users/aldous/RWG/book.html.

[2] R. Aleliunas, R.M. Karp, R.J. Lipton, L. Lovász and C. Rackoff, Random Walks, Universal Traversal Sequences, and the Complexity of Maze Problems. Proceedings of the 20th Annual IEEE Symposium on Foundations of Computer Science (1979) 218-223.

[3] C. Avin and G. Ercal, On the cover time and mixing time of random geometric graphs, Theoretical Computer Science 380 (2007) 2-22

[4] K. Ball, An elementary introduction to modern convex geometry, in Flavors of Geometry, MRI Publication 31, S. Levy Ed. (1997) 1-58.

[5] Z. Bar-Yossef, R. Friedman and G. Kliot, RaWMS - Random Walk based Lightweight Membership Service for Wireless Ad Hoc Networks, TOCS 2008, MOBIHOC 2006.

[6] S.Boyd, A. Ghosh, B. Prabhakar and D. Shah, Mixing Times for RandomWalks on Geometric Random Graphs, In Proceedings of the 2nd SIAM Workshop on Analytic Algorithmics and Combinatorics (ANALCO) (2005).

[7] J. Brown and R. Churchill, Complex Variables and Applications, (Sixth Edition) McGrawHill (1996).

[8] C. Cooper and A. M. Frieze, The cover time of random regular graphs, SIAM Journal on Discrete Mathematics, 18 (2005) 728-740.

[9] C. Cooper and A. M. Frieze, The cover time of the preferential attachment graph. Journal of Combinatorial Theory Series B, Series B, 97(2) 269-290 (2007).

[10] C. Cooper and A. M. Frieze, The cover time of sparse random graphs, Random Structures and Algorithms 30 (2007) 1-16.

[11] C. Cooper and A. M. Frieze, The cover time of the giant component of a random graph, Random Structures and Algorithms, 32, 401-439 (2008).

[12] C. Cooper and A. M. Frieze, The cover time of random digraphs, Proceedings of RANDOM 2007, (11th. International Workshop on Randomized Techniques in Computation)

[13] P. G. Doyle and J. L. Snell, Random Walks and Electrical Networks, Carus Mathematical Monograph 22, AMA (1984).

[14] R. Durrett, Probability: Theory and Examples, Brooks/Cole 1991.

[15] U. Feige, A tight upper bound for the cover time of random walks on graphs, Random Structures and Algorithms, 6 (1995) 51-54. 
[16] U. Feige, A tight lower bound for the cover time of random walks on graphs, Random Structures and Algorithms, 6 (1995) 433-438.

[17] W. Feller, An Introduction to Probability Theory, Volume I,(Second edition) Wiley (1960).

[18] P. Gupta and P.R. Kumar, Critical power for asymptotic connectivity in wireless networks, In Stochastic Analysis, Control, Optimization and Applications, Birkhaüser, Boston, 1998.

[19] P. Gupta and P.R. Kumar, Internets in the sky: the capacity of three dimensional wireless networks. Communications in Information and Systems, 1 (2001) 33-50.

[20] L. Lovász. Random walks on graphs: a survey. Bolyai Society Mathematical Studies, Combinatorics, Paul Erdös is Eighty (Volume 2) Keszthely (Hungary), (1993) 1-46.

[21] M. D. Penrose. Random Geometric Graphs. Oxford University Press (2003).

[22] A. Sinclair. Improved bounds for mixing rates of Markov chains and multicommodity flow. Combinatorics, Probability and Computing, 1 (1992), 351-370.

[23] H. Wilf. Generatingfunctionology, Academic Press (1990).

[24] F. Xue and P. R. Kumar, Scaling laws for ad hoc wireless networks: an information theoretic approach. Foundations and Trends in Networking, 1(2) (2006) 145-270.

\section{A Proof of $(36)$}

Fix two vertices $u, v$. For $t \geq T$ let $h_{t}=\operatorname{Pr}\left(\mathcal{W}_{u}(t)=v \mid \mathcal{B}_{v}\right)$ be the conditional probability that the walk $\mathcal{W}_{u}$ visits $v$ at step $t$. Let

$$
H(z)=\sum_{t=T}^{\infty} h_{t} z^{t}
$$

generate $h_{t}$ for $t \geq T$.

Next, considering the walk $\mathcal{W}_{v}$, starting at $v$, let $r_{t}=\operatorname{Pr}\left(\mathcal{W}_{v}(t)=v\right)$ be the probability that this walk returns to $v$ at step $t=0,1, \ldots$ Let

$$
R(z)=\sum_{t=0}^{\infty} r_{t} z^{t}
$$

generate $r_{t}$. Our definition of return involves $r_{0}=1$. 
For $t \geq T$ let $f_{t}=f_{t}(u \rightarrow v)$ be the probability that the first visit of the walk $\mathcal{W}_{u}$ to $v$ in the period $[T, T+1, \ldots]$ occurs at step $t$, conditional on the occurrence of $\mathcal{B}_{v}$. Let

$$
F(z)=\sum_{t=T}^{\infty} f_{t} z^{t}
$$

generate $f_{t}$. Then we have

$$
H(z)=F(z) R(z)
$$

Write

$$
R(z)=R_{T}(z)+\widehat{R}_{T}(z)+\frac{\pi_{v} z^{T}}{1-z},
$$

where $R_{T}(z)$ is given by $(10)$ and

$$
\widehat{R}_{T}(z)=\sum_{t \geq T}\left(r_{t}-\pi_{v}\right) z^{t}
$$

generates the error in using the stationary distribution $\pi_{v}$ for $r_{t}$ when $t \geq T$. Similarly,

$$
H(z)=\widehat{H}_{T}(z)+\frac{\pi_{v} z^{T}}{1-z}
$$

where

$$
\widehat{H}_{T}(z)=\sum_{t \geq T}\left(h_{t}-\pi_{v}\right) z^{t}
$$

Equation (9) implies that the radii of convergence of both $\widehat{R}_{T}$ and $\widehat{H}_{T}$ exceed $1+2 \lambda$. (Although the $h_{t}$ are conditional probabilities, we can still use (9) for $\left.t \geq 2 T\right)$. Moreover, $|z| \leq 1+\lambda$,

$$
\begin{aligned}
& \left|\widehat{R}_{T}(z)\right|=o\left(n^{-2}\right) . \\
& \left|\widehat{H}_{T}(z)\right|=o(1) .
\end{aligned}
$$

Equation (55) follows directly from the definition of $T$. This also implies that

$$
\sum_{t \geq 2 T}\left|h_{t}-\pi_{v}\right||z|^{t}=o\left(n^{-2}\right)
$$

Furthermore, $\sum_{t=T}^{2 T} \pi_{v}|z|^{2 T}=O\left(T \pi_{v}\right)=o(1)$ and

$$
\sum_{t=T}^{2 T} h_{t}|z|^{t} \leq(1+\lambda)^{2 T} \sum_{t=T}^{2 T} h_{t} \leq(1+\lambda)^{2 T} \operatorname{Pr}\left(\mathcal{B}_{v}\right)^{-1} \max _{u^{\prime}} R_{u^{\prime}, v}=o(1)
$$

where for $u^{\prime} \neq v$ we let $R_{u^{\prime}, v}$ denote the expected number of visits by $\mathcal{W}_{u}$ to $v$ up to time $T$.

Here $\operatorname{Pr}\left(\mathcal{B}_{v}\right)^{-1}$ handles the conditioning on $\mathcal{B}_{v}$ and maximising over $u^{\prime}$ is maximising over $\mathcal{W}_{u}(T-1)$. 
Using (53), (54) we rewrite $F(z)=H(z) / R(z)$ from (52) as $F(z)=B(z) / A(z)$ where

$$
\begin{aligned}
& A(z)=\pi_{v} z^{T}+(1-z)\left(R_{T}(z)+\widehat{R}_{T}(z)\right), \\
& B(z)=\pi_{v} z^{T}+(1-z) \widehat{H}_{T}(z) .
\end{aligned}
$$

For real $z \geq 1$ we have

$$
R_{T}(1) \leq R_{T}(z) \leq R_{T}(1) z^{T}
$$

Let $z=1+\beta \pi_{v}$, where $\beta=O(1)$. Since $T \pi_{v}=o(1)$ we have

$$
R_{T}(z)=R_{T}(1)\left(1+O\left(T \pi_{v}\right)\right) .
$$

$T \pi_{v}=o(1)$ and $T \pi_{v}=\Omega\left(n^{-2}\right)$ and (55) and $R_{T}(1) \geq 1$ imply that

$$
A(z)=\pi_{v}\left(1-\beta R_{T}(1)+O\left(T \pi_{v}\right)\right)
$$

It follows that $A(z)$ has a real zero at $z_{0}$, where

$$
z_{0}=1+\frac{\pi_{v}}{R_{T}(1)\left(1+O\left(T \pi_{v}\right)\right)}=1+p_{v}
$$

say. We also see that

$$
A^{\prime}\left(z_{0}\right)=-R_{T}(1)\left(1+O\left(T \pi_{v}\right)\right) \neq 0
$$

and thus $z_{0}$ is a simple zero (see e.g. [7] p193). The value of $B(z)$ at $z_{0}$ is

$$
B\left(z_{0}\right)=\pi_{v}(1+o(1)) \neq 0
$$

We have used (56) here.

Thus,

$$
\frac{B\left(z_{0}\right)}{A^{\prime}\left(z_{0}\right)}=-\left(1+O\left(T \pi_{v}\right)\right) p_{v} .
$$

Thus (see e.g. [7] p195) the principal part of the Laurent expansion of $F(z)$ at $z_{0}$ is

$$
f(z)=\frac{B\left(z_{0}\right) / A^{\prime}\left(z_{0}\right)}{z-z_{0}}
$$

To approximate the coefficients of the generating function $F(z)$, we now use a standard technique for the asymptotic expansion of power series (see e.g.[23] Th 5.2.1).

We prove below that $F(z)=f(z)+g(z)$, where $g(z)$ is analytic in $C_{\lambda}=\{|z| \leq 1+\lambda\}$ and that

$$
M=\max _{z \in C_{\lambda}}|g(z)|=O\left(T \pi_{v}\right) .
$$


Let $a_{t}=\left[z^{t}\right] g(z)$, then (see e.g. [7] p143), $a_{t}=g^{(t)}(0) / t$ !. By the Cauchy Inequality (see e.g. [7] p130) we see that $\left|g^{(t)}(0)\right| \leq M t ! /(1+\lambda)^{t}$ and thus

$$
\left|a_{t}\right| \leq \frac{M}{(1+\lambda)^{t}}=O\left(T \pi_{v} e^{-t \lambda / 2}\right) .
$$

As $\left[z^{t}\right] F(z)=\left[z^{t}\right] f(z)+\left[z^{t}\right] g(z)$ and $\left[z^{t}\right] 1 /\left(z-z_{0}\right)=-1 / z_{0}^{t+1}$ we have

$$
\left[z^{t}\right] F(z)=\frac{-B\left(z_{0}\right) / A^{\prime}\left(z_{0}\right)}{z_{0}^{t+1}}+O\left(T \pi_{v} e^{-t \lambda / 2}\right) .
$$

Thus, we obtain

$$
\left[z^{t}\right] F(z)=(1+o(1)) \frac{p_{v}}{\left(1+p_{v}\right)^{t+1}}+O\left(T \pi_{v} e^{-t \lambda / 2}\right) .
$$

Substituting $R_{T}(1)=1+o(1)$ and $\pi_{v} \sim \frac{c-1}{c n}$ completes the proof of (36).

\section{A.1 Proof of (64)}

Now $M=\max _{z \in C_{\lambda}}|g(z)| \leq \max |f(z)|+\max |F(z)|=O\left(T \pi_{v}\right)+\max |F(z)|$, where $F(z)=$ $B(z) / A(z)$. On $C_{\lambda}$ we have, using (55)-(58),

$$
|F(z)| \leq \frac{O\left(\pi_{v}\right)}{\lambda\left|R_{T}(z)\right|-O\left(T \pi_{v}\right)}=O\left(T \pi_{v}\right) .
$$

We now prove that $z_{0}$ is the only zero of $A(z)$ inside the circle $C_{\lambda}$ and this implies that $F(z)-f(z)$ is analytic inside $C_{\lambda}$. We use Rouché's Theorem (see e.g. [7]), the statement of which is as follows: Let two functions $\phi(z)$ and $\gamma(z)$ be analytic inside and on a simple closed contour C. Suppose that $|\phi(z)|>|\gamma(z)|$ at each point of $C$, then $\phi(z)$ and $\phi(z)+\gamma(z)$ have the same number of zeroes, counting multiplicities, inside $C$.

Let the functions $\phi(z), \gamma(z)$ be given by $\phi(z)=(1-z) R_{T}(z)$ and $\gamma(z)=\pi_{v} z^{T}+(1-z) \widehat{R}_{T}(z)$.

$$
|\gamma(z)| /|\phi(z)| \leq \frac{\pi_{v}(1+\lambda)^{T}}{\lambda \theta}+\frac{\left|\widehat{R}_{T}(z)\right|}{\theta}=o(1) .
$$

As $\phi(z)+\gamma(z)=A(z)$ we conclude that $A(z)$ has only one zero inside the circle $C_{\lambda}$. This is the simple zero at $z_{0}$. 